\title{
Quality Assurance of Bottling Plant Using Image Processing and PLC
}

\author{
Radhakrishnan $\mathrm{K}^{1}$, Neeraja $\mathrm{V}^{2}$ \\ ${ }^{1}$ (HOD Electronics and Communication HKCET, Pampakuda, Ernakulam, India) \\ ${ }_{2}^{2}$ (Student Department of Electrical and Electronics HKCET, Pampakuda, Ernakulam, India)
}

\begin{abstract}
Image processing based quality checking is one of the most popular technologies employed in manufacturing process nowadays, as it reduces both the human errors and the man power requirement. The purpose of this project is to identify the external defects of the bottle/can which are manufactured in an industry and to eliminate such products to assure the quality of the final products. Main objective of this project is to identifying the defective objects which arrive through conveyer lines using a specialized camera which is controlled by a PC. Image processing using Raspberry Pi is used for processing the images which is very user friendly. The bottle/ can moves through a conveyor. A proximity sensor will sense the object and stop the conveyor. Then the image of the object will be taken by the camera and compare the image with the already stored template image using Raspberry Pi. Object that falls within the prescribed tolerance level be regarded as good object. The conveyor will take the bottle/ can to the filling section and others will discarded. Bad bottle/can will be removed with the help of a solenoid piston. The conveyor control, sensing operation, removal of defective piece all are controlled by PLC. This kind of a system is very less in cost comparing to the other image processing quality control systems which are used in industries.
\end{abstract}

Keywords: Raspberry Pi, PLC, image processing, template image

\section{Introduction}

In large scale manufacturing plants thousands of products are manufactured every day. Due to errors in the manufacturing process, damaged or under qualified products are unavoidable. Rejecting such products before supplying to the customer becomes essential. Quite often especially in small scale process this operation is done manually. Even though the manual method suits some small scale organizations; this doesn't suite large manufacture plants. Due to heavy work load humans operators may lack in performing the work with a high accuracy. Even efficiency will fall down. Just for this operation the company will have to arrange special quality control workers where in a large company it will end up being costly. Further this requires high manpower and time. Inefficiency of the quality control system can affect the company in large scale product rejection. This paper presents a system which will do the quality control operation in bottle/can manufacturing process with higher precision accuracy by rejecting the defective ones. It also aims to maintaining a data base to have details about the final rejected products which can be used for modifying the production process in future. The section 2 provides information about the exiting works. Section 3 discusses the proposed system. The experimental set up and results are discussed in section 4 .The section 5 concludes the paper.

\section{Existing Works}

[1] K.S.Shilpashree1 etc"Implementation of Image Processing on Raspberry Pi". This paper presents the implementation of image processing operations on Raspberry Pi. Here this concept is used in the real time application of micro air vehicle (MAV), The MAVs are basically used to capture images and videos through the Raspberry pi camera module. [2].G.Senthilkumar1 etc "Embedded Image Capturing System Using Raspberry Pi system " This paper proposed an image capturing technique in an embedded system based on Raspberry Pi board.Experimental results show that the designed system is fast enough to run the image capturing, recognition algorithm, and the data stream can flow smoothly between the camera and the Raspberry Pi board. [3].Edwin Jose Kundukulam etc "Implementing and Optimizing Template Matching Techniques for Home Automation". In this paper, the concept of object recognition using Raspberry Pi is proposed for home automation using template matching. The algorithms used were SURF and squared difference error method algorithm. Home automation is done based on template matching. [4] Varshal Shah etc "Image Processing Based Bottle Filling and Label Checking Using Embedded System". This paper aims to present image processing techniques used for automatic bottle filling and label checking.In this system various image processing techniques are used for liquid level detection such as image filtering, image thresholding, image segmentation, hole filling algorithm, contour fitting. 


\section{Proposed System}

In this project we have developed a system which will do the quality control operation in an automated manner by measuring the dimensions of final product and reject the products with defects. It also aims to maintaining a data base to have details about the final rejected products which can be used for modifying the production process in future. First the bottle/can passes through the conveyor belt. Then it is identified by the sensor. Proximity- Sensors have been selected to sense the presence of the objects which arrive in the conveyer line for inspection.. The conveyor will be stopped when object reached a camera unit. During that process, the camera will be ON and suddenly capture the image of current object. Then the camera will send the captured image to the Raspberry Pi. The software will compare the original image (required design and size) and the current image. The original image is previously stored in the software. If the compared image is good then the conveyor will move and go to filling process and restart the process by taking another set of bottle/can.If the specimen does not meet the specifications it will be treated as a defective one. The system will allow the operator toremove the defective object and it does not happen within 10 seconds. The PLC actuate a solenoid piston which will automatically remove the defective piece from the conveyor line .Thus the process will restart, by taking the next piece of bottle/can. Fig: 1 gives the idea about the proposed system architecture. System can be classified in three groups, one is power circuitry, second one is controller sections and the third one is sensor sections.

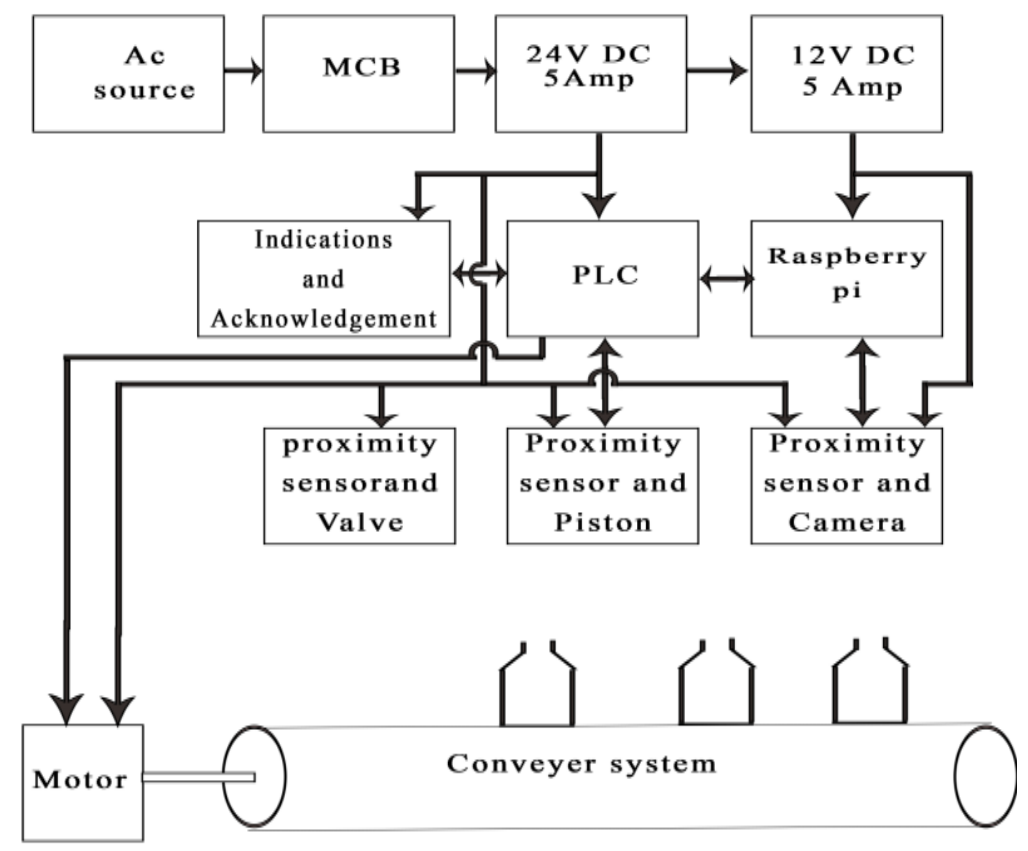

Fig: 1 Proposed System Architecture

\section{Experimental Set Up And Working}

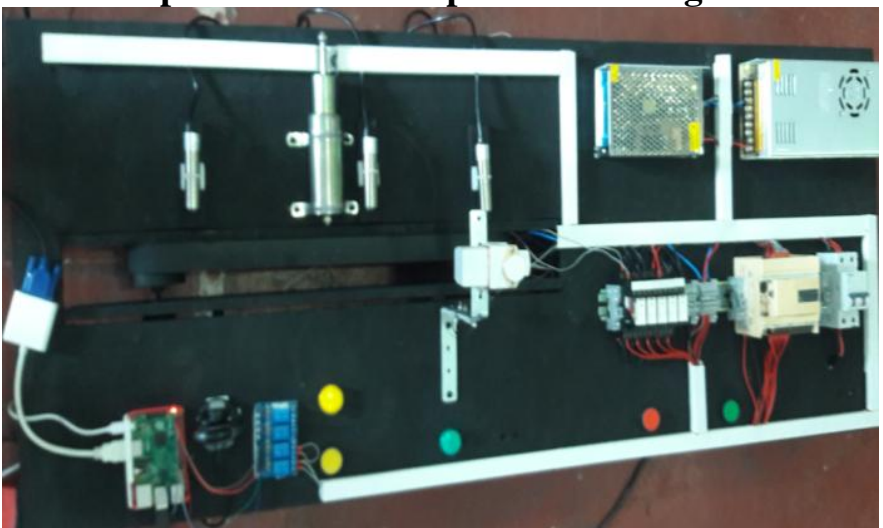

Fig:2 Prototype 
Components used in this prototype can be classified in three groups, one is power circuitry, second one is controller sections and the third one is sensor sections. Sensory section consists of proximity sensors panel, camera panel, solenoid piston and conveyor lines. Proximity sensor panel: This panel consists of transmitter and a receiver. When the object passes the panel the voltage level between the transmitter and receiver becomes low. This panel is used to sense the presence of the objects/ products in the conveyer line. Photo sensors, type: PNP, sensing range: $\mathrm{NC}$, outer diameter: $\mathrm{NO}+\mathrm{NC}$, operating voltage:6-36 v dc. Camera Panel: Once the presence of the object is sensed the camera panel will be activated. The camera will take the picture of the object and the PC interface will analyze the picture to find deformed parts in the object. Camera used is I ball chd20web cam with resolution 20MP(photo),2.1(video) and image resolution of 5500*3640pixelsFrames per second:30. Solenoid piston: This will be activated when PC interface finds faults in the picture/image. And the actuator will move the deformed products to the bin or another moving conveyer line. $24 \mathrm{v}$ dc, $8 \mathrm{~A}-20 \mathrm{~A} \mathrm{~cm}$ shaft length,5cm displacement. Conveyer Lines/ Bin: If there is no faults in the product it will keep moving on the conveyer line for filling or will be moved to a final product bin. If there are faults in the products they will be pushed to from conveyer line or a waste bin. Raspberry pi board: The Raspberry Pi is a basic embedded system having a credit card-sized single board computers developed in the UK by the Raspberry Pi Foundation The Raspberry Pi board is the central module of the whole embedded image capturing and processing system as given in fig. 2. Its main parts include: main processing chip unit, memory, power supply HDMI Out i.e VGA display, Ethernet port, and USB ports. 6PLC:Schineider electrical TWIDO is the brand-name of a line of factory automation equipment manufactured by Schineider electric company. Model no:TWD LCDA 24DRF,Communication port-MD8,PLC used is without smps, module expandable upto $4 \mathrm{I} / \mathrm{O}$ modules. software used is TWIDO suite. The conveyor control, sensing operations, diversion control and etc. each operation should be controlled by PLC. The PLC is one of the controller modules which can control the output devices with the help of input devices and developed a program. Power circuitry parts consists of the following elements dc motor, smps, internal and external relays. DCmotor:24 v dc motor with $100 \mathrm{rpm}$ which is easily available in market. This motor is used in our project for the movement of conveyor. Internal relays:4 channel SPDT type internal relays are used in our project.5 v dc,optocouplertype. We are using two channels out of 4 , one for activate good piece and other for bad piece. SPDT means single pole double through switches External relay:24vdc,DPDT type is used in our project. Company name is o/e/n. DPDT means double pole double through switches. External relays help us to isolate PLC from other power circuitries. Switching mode power supply ,it is an electronic power supply that incorporates a switching regulator to convert electric power efficiently. Like other power supply an SMPS transfers power from a dc or a ac source. Voltage regulation is achieved by varying the ratio o on- to- off time. The higher power conversion efficiency is an advantage of SMPS. Here in this project 2 SMPS are used,24 V

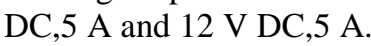
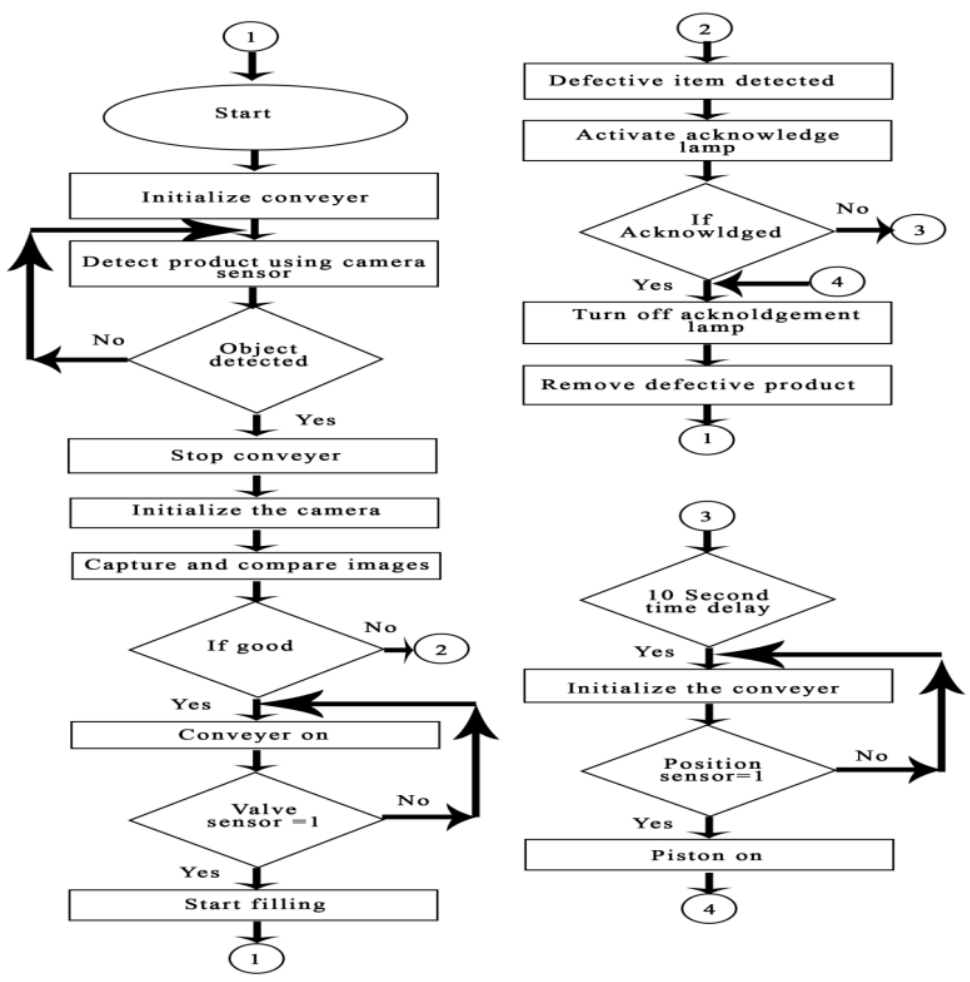

Fig. 3 Process Flow Chart 
In this paper quality checking of a bottling plant has been implemented using PLC and Raspberry Pi. Image processing technique is one of the effective tool for finding the quality of a product Here a bottle /can be move through a conveyor.A proximity sensor will sense the object and stop the conveyor. The camera capture the image of the object and compares the template using the Raspberry Pi. A reasonably good match be considered as a good object. The conveyor will take qualified bottle/can to the filling section and others will be treated as bad objects .Bad object /bottle can be removed with the help of solenoid piston. The conveyor control, sensing operations, removal of defective piece all should be controlled by PLC.

Image Processingusing Raspberry PI: It's a process of realizing embedded image capturing system. A design of the experimental prototype of the embedded image capturing system with Raspberry $\mathrm{Pi}$ has been implemented. This system is smaller, lighter and with lower power consumption, so it is more convenient than the other image processing methods. Because of the open source code, it is freer to do software development on Linux. Experimental results show that it's an effective method of using Raspberry Pi board to realize on embedded image capturing system. In this system raspberry pi is used so it reduce the need of computer. Due to this cost of the system is reduced. In future it is also possible to inspect faulty bottle and cap position of bottle using image processing techniques The Raspberry Pi board is the central module of the whole embedded image capturing and processing system. Steps involved in image processig using raspberry pi: First install the raspberry pi OS to the SD card, then connect components to the hardware and switch on the supply. Login the raspberry pi board using by username and password. Check the network settings, enable the camera and capture an image ,run the code in python .Check the enhancement algorithms, this help us to remove noise present in aimage.Image processing using raspberry can be done with the help of simple CV, it is a library which help us to perform complicated image manipulation task very easily. First connect the camera module,then check the camera is connected ,then install simple CV after that load an image from the camera into the simple CV. Then we can perform the image processing. Enhancements method or algorithms such as template matching techniques are used.Image processing start with the import of libraries of simpleCV.Then initialize the camera and GPIO. The object is detected using sensor and image is captured by the camera. If no object is detected,print nil.Load the image from the camera into the simpleCV.Compare captured image with template.If the image matches with the template image then it is considered as good image. Then initiate display good on the monitor.If the image does not match with the template image,then image is considered as bad image and display bad.This process flow chart is depicted in fig. 4 .

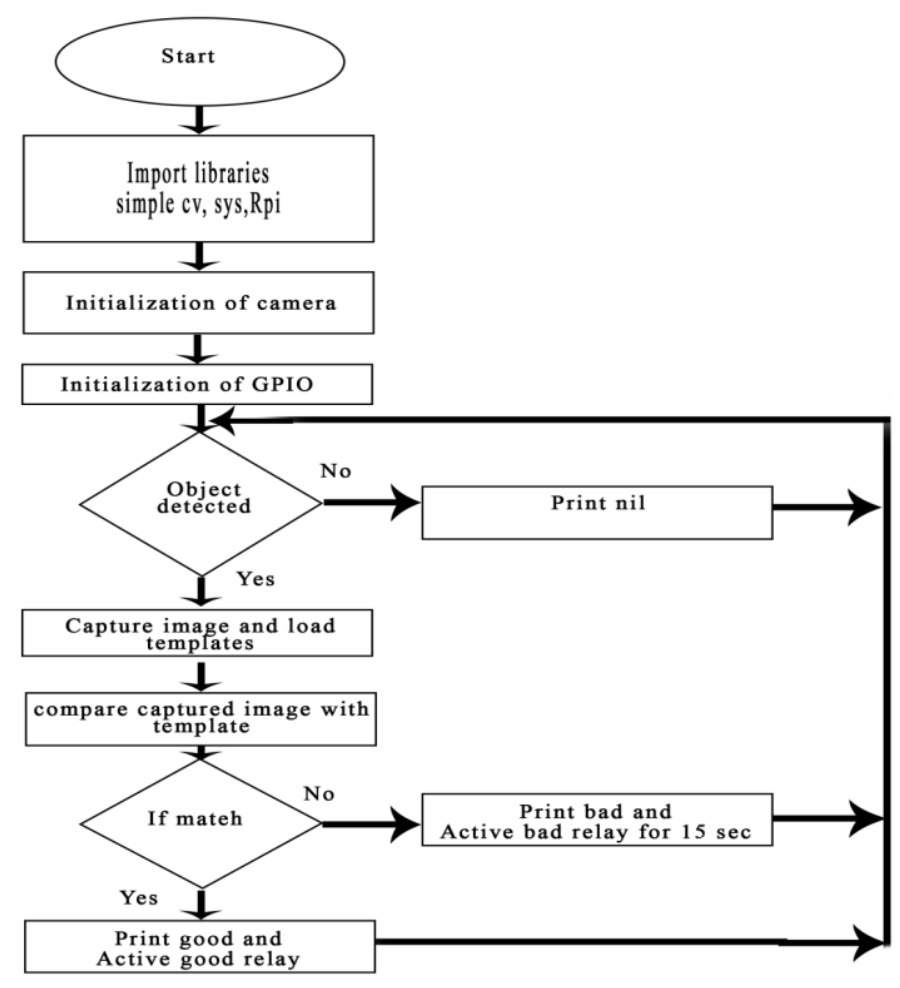

Fig 4 Image processing flow chart 
Image processing based quality checking is one of the most popular technologies because it reduces the human errors and also reduce the workforce of human. In this project comparison of different template matching methods are also done. Fig.2 shows the prototype developed.The developed system has been tested thrice consisting of 15 samples in each batch. The methods adopted for testing are Normalized square difference matching method (CV_TM_SQDIFF_NORMED), Normalized correlation matching method (CV_TM_CCORR_NORMED), third one is Normalized correlation coefficient matching method (CV_TM_CCOEFF_NORMED).The table 1 compares the percentage good and bad images for the 3 methods. The normalized methods are used because it can help to reduce the effects of illumination differences of template and the images. Comparing the chart with different normalized template methods, we understand that using square difference method perfect match is almost zero, imperfect match is large. So we have adapted the second method that is the correlation method .Using this method we are getting more matches. Less number of defective match .but getting more matches means almost similar to template image are taken as good image .Using this method, accuracy is less .So we continue our trials with the third method that is correlation method, which is more sophisticated than the simple square difference method, using this method we get perfect matches and also imperfect matches .It also having very good accuracy. So we suggest correlation coefficient is best out of three methods. This values can be used in statistical process control (SPC).

Table: 1 comparison Table With Different Template Methods

\begin{tabular}{|c|c|c|}
\hline Methods & \multicolumn{2}{|c|}{ Average of 3 trials each with a batch of 15 } \\
\cline { 2 - 3 } & GOOD IMAGES & BAD IMAGES \\
\hline $\begin{array}{c}\text { Normalized square difference matching } \\
\text { method(CV_TM_SQDIFF_NORMED) }\end{array}$ & $8.88 \%$ & $91.11 \%$ \\
\hline $\begin{array}{c}\text { Normalized correlation matching } \\
\text { method(CV_TM_CCORR_NORMED) }\end{array}$ & $91.11 \%$ & $8.88 \%$ \\
\hline $\begin{array}{c}\text { Normalized correlation coefficient } \\
\text { matchingmethod(CV_TM_CCOEFF_NORMED) }\end{array}$ & $75.55 \%$ & $24.44 \%$ \\
\hline
\end{tabular}

\section{Conclusion}

This project addresses the design and implementation of a quality control scheme for a bottle/can manufacturing process. It makes use of the decision making/timing control characteristics of PLC and simple but efficient embedded image capturing of a Raspberry Pi system. Image processing based quality assurance system is becoming one of the important factors in the modern industries in order to make the products more perfect and reduce the errors by the human workforce. Here a Logitech high definition camera has been programmed with Raspberry Pi for inspecting the products, which deliver from the manufacturing plant. A Programmable Logic controller with limited inputs and outputs is connected through a serial interface to the PC interface which helps fast data transaction between the PC interface and other components such as sensors, solenoid piston and other external components as network and displays.

\section{References}

[1] BAUMER SENSORS, Photoelectric sensors for countless applications http://www.baumer.com viewed January 2016

[2] BIRENDER SINGH et al, Removal of Defective Products Using Robots, Dept. of Electronics and Communication Engineering, BIT, Mesra, India 2013

[3] CELSON LIMA et al, MATLAB framework for PLC controlled Processes, College of Industrial engineering Barcelona, $<$ http://www. Intechopen.com> viewed February 2016

[4] CHITRA.S et al, Department of Electronics and Communications Engineering, Advancements in Research \& Technology, Volume 3, Issue 8, August-2014

[5] CVG COMMERCIAL VEHICLE GROUP, Supplier Quality and Packaging Requirements Manual, New Albany Ohio, 2012

[6] K.S.Shilpashree1, Lokesha.h, HadimaniShivkumar" Implementation of Image Processing on Raspberry Pi"

[7] G.Senthilkumar1, K.Gopalakrishnan, V. Sathish Kumar "Embedded Image Capturing System Using Raspberry Pi system

[8] Edwin Jose Kundukulam and Abraham Sudharson "Implementing and Optimizing Template Matching Techniques for Home Automation" 